\title{
Diffusion tensor imaging of the superior cerebellar peduncle
}

\author{
Bilal Battal • Salih Hamcan • Veysel Akgun
}

Received: 25 July 2013 / Accepted: 7 August 2013 /Published online: 18 August 2013

(C) Springer-Verlag Berlin Heidelberg 2013

\section{Dear Editor:}

We read the recent article titled "Diffusion tensor imaging of the superior cerebellar peduncle identifies patients with posterior fossa syndrome" by Ojemann et al. [2], published online in Child's Nervous System (doi: 10.1007/s00381-013-2205-6), with great interest. The authors evaluated the involvement of the dentothalamic tracts, specifically the superior cerebellar peduncle (SCP), in patients with posterior fossa tumors and the association with posterior fossa syndrome (PFS) using diffusion tensor imaging (DTI). We believe that this article will be a useful DTI guide for radiologists and clinicians in assessing the integrity of SCP and in predicting the occurrence probability of PFS. We believe that the readers who are experts on DTI and fine radiologic brain anatomy have not experienced any problems in understanding this top-quality and interesting paper. Because the readers of the Child's Nervous System are surgeons and pediatric clinicians in addition to radiologists, we need to clarify a few technical points about the DTI and fractional anisotropy (FA) map in order not to cause misunderstandings in readers who are newly familiar to this advanced magnetic resonance imaging technique.

First, the definition of the SCP on colored FA map images may cause some confusion in some readers. The authors defined the superior cerebellar peduncle as a blue color in the caption of Fig. 1 and indicated with arrows on colored FA map images. SCP is a mixture of green and blue (turquoise, light blue, or greenish blue) on colored FA maps due to its oblique (some horizontal and some vertical) orientation as also described by the authors. Actually, the color of the more horizontal cerebellar portion of SCP is closer to green, and the color of the more vertical brainstem portion is closer to blue on colored

B. Battal $(\bowtie) \cdot$ V. Akgun

Department of Radiology, Gulhane Military Medical School,

06018 Etlik, Ankara, Turkey

e-mail: bilbat_23@yahoo.com

S. Hamcan

Department of Radiology, Balikesir Military Hospital,

Balikesir, Turkey
FA maps. Moreover, the arrows on Fig. 1 are a little too distant from the SCP, and these arrows may be wrongly perceived to point to another adjacent structure which is coded in dark blue color. We think these two issues may be a cause of misunderstanding in the readers especially in those newly familiar to relatively low-spatial-resolution FA maps.

Second, we want to emphasize that colors of white matter tracts on FA maps are strictly dependent on their course. As also described by the authors, color is used to specify the principal diffusion direction in each voxel (typically red representing left-right anisotropy; green, anterior-posterior; and blue, superior-inferior) on colored FA maps [2]. Diffusion in oblique directions such as SCP is coded by a precisely proportional mixture of the three principal colors (blue, green, and red) [1]. The intensity of the color is proportional to the FA value. If a tumor compresses the superior cerebellar peduncle superiorly, anteriorly, or laterally and causes a change in its oblique (some horizontal, some vertical) orientation, SCP may be coded with a different color such as blue, green, and even closer to red due to the different orientation on colored FA maps. Because of this, to seek a standard color (mixture of green and blue) of SCP on FA maps for the evaluation of its integrity may be misleading, especially in SCP compressed by a large tumor. We believe that the evaluation of high-resolution and detailed anatomical T1- and T2-weighted images beside FA maps is crucial for evaluation of the integrity of SCP.

Conflict of interest The authors declare that they have no conflict of interest.

\section{References}

1. Naidich TP, Castillo M, Cha S, Smirniotopoulos SG (2012) Imaging of the brain. In: Naidich TP, Krayenbuhl H, Kollias S, Bou-Hidar P, Bluestone AY, Carpenter DM (eds) White matter, 1st edn. Elsevier/ Saunders, Philadelphia, pp 205-244

2. Ojemann JG, Partridge SC, Poliakov AV, Niazi TN, Shaw DW, Ishak GE, Lee A, Browd SR, Geyer JR, Ellenbogen RG (2013) Diffusion tensor imaging of the superior cerebellar peduncle identifies patients with posterior fossa syndrome. Childs Nerv Syst. doi:10.1007/s00381-013-2205-6 